\title{
External validity of randomised controlled trials in idiopathic pulmonary fibrosis
}

\section{To the Editors:}

I would like to comment on the editorial by JOHNSON and RAGHU [1], which was published in the November issue of the European Respiratory Journal. In a very clear way, the authors overviewed the use of outcome measures in clinical trials of idiopathic pulmonary fibrosis (IPF).

I would like to propose some concerns, which could affect the external validity of randomised controlled trials in IPF [2, 3]. The first concern is about the diagnostic accuracy of the disease. IPF is a rare disease and no single accurate test for the diagnosis of IPF exists. Studies of the accuracy of diagnosing IPF are performed mostly in tertiary referral centres, and, even in these studies, an important interobserver variability exists. In most of these studies, prior knowledge of the presence of a form of interstitial lung disease existed, which may evoke an observer bias and, therefore, influence the results on the diagnostic accuracy [3].

The incidence of IPF in a general pulmonary practice is low. Diagnostic accuracy (i.e. sensitivity and specificity) also depends on the prevalence of the disease. A lower prevalence of the disease results in a higher number of false-positive and false-negative diagnoses [3]. In recent published trials, participating centres were selected from tertiary care centres or secondary care centres with particular interests in the management of IPF.

If a treatment for idiopathic pulmonary fibrosis is found, the chance that this treatment will be given to patients without idiopathic pulmonary fibrosis is high in a pulmonary care practice with a very low incidence of idiopathic pulmonary fibrosis. Or to summarise these concerns using a question: will it be possible to generalise these trials in a general pulmonary care practice [2]?

\section{Thomeer}

Respiratory Medicine, UZ Leuven, Leuven, Belgium.

\section{REFERENCES}

1 Johnson WC, Raghu G. Clinical trials in idiopathic pulmonary fibrosis: a word of caution concerning choice of outcome measures. Eur Respir J 2005; 26: 755-758.

2 Rothwell PM. External validity of randomised controlled trials: "to whom do the results of this trial apply?". Lancet 2005; 365: 82-93.

3 Knottnerus JA, van Weel C, Muris JW. Evaluation of diagnostic procedures. BMJ 2002; 324: 477-480.
From the authors:

We appreciate the important concern raised by $\mathrm{M}$. Thomeer and agree that following a standardised diagnostic criteria is important for both the investigator as well as the clinician taking care of the patients in the community. While the general pulmonologist may not be as "accurate" in diagnosing idiopathic pulmonary fibrosis (IPF) as expert clinicians involved in the diagnosis and management of IPF and other subgroups of idiopathic interstitial pneumonias (IIP) in tertiary centres enrolling patients in clinical trials, the clinical knowledge of IPF has evolved significantly with new data and clarifications during the last few years. Recent international consensus statements by the American Thoracic Society (ATS) and the European Respiratory Society (ERS) have increased the awareness of the different entities of IIP and IPF [1, 2]. Thus, the current generation of general pulmonologists, as well as experienced pulmonologists, involved in active care of patients with pulmonary diseases are becoming quite familiar with the diagnostic criteria for these entities. In fact, all of the recent and ongoing clinical trials have utilised well-defined criteria based on set guidelines to enrol the most appropriate patients. The striking similarity of the baseline demographics in the patient populations enrolled in two recent separate multicentre clinical trials in the USA, Canada and Europe illustrates this very well $[3,4]$.

Indeed, results from a trial apply to the population from which the trial sample is drawn. It should be noted that the patients enrolled in recent IPF clinical trials do come from the community, the majority of whom are referred by general pulmonologists to centres conducting clinical trials in IPF. If the diagnosis criteria utilised by general pulmonologists (and, therefore, the "IPF population in the community") is drastically different from that utilised for the IPF sample enrolled in the clinical trials, we would agree that the results from the trial may not be relevant to the IPF patients cared for by general pulmonologists. This would be an appropriate concern if patients were simply enrolled in trials without well-defined eligibility criteria. Enrolling patients with well-defined inclusion and exclusion criteria is the only means of assuring the appropriate study population pertinent to the clinical trial. It must also be noted that in the largest IPF clinical trial reported to date, general pulmonologists in clinical practice, without a previous track record as "IPF/interstitial lung disease experts", directly enrolled the IPF patients to the trial. The patients, from several centres in the USA, Canada and Europe, were enrolled by clinicians who followed the study protocols in their own clinics (i.e. not necessarily in the tertiary and secondary centres) quite well [3]. The ongoing International study of Survival outcomes in idiopathic Pulmonary fibrosis with InteRfEon gamma trial has enrolled 800 patients from several sites in the USA, Europe and 